This is the author's version of an article that has been published in the ICIT 2019 proceedings.

Changes were made to this version by the publisher prior to publication.

The final version of record is available at https://dx.doi.org/10.1109/ICIT.2019.8755017

\title{
Investigation on an AC Grid Failure Handling of Industrial DC Microgrids with an Energy Storage
}

\author{
Alexander Männel \\ Bosch Rexroth AG \\ Lohr am Main, Germany \\ Email: Alexander.Maennel@boschrexroth.de
}

\author{
Svenja Tappe, Elias Knöchelmann, Tobias Ortmaier \\ Gottfried Wilhelm Leibniz Universität Hannover \\ Institute of Mechatronic Systems \\ Hannover, Germany
}

\begin{abstract}
A new approach for more energy efficient industrial production processes are smart industrial direct current (DC) microgrids with one or more connections to the alternative current (AC) grid. The advantage of the DC-technology is an easier integration of renewable energies sources and energy storage systems (ESS). Different applications for ESS are possible, for instance an uninterruptible power supply (UPS) for a DC microgrid. Within this paper, a new handling concept for a mains supply failure (e.g. a blackout of the supplying AC grid) with a droop curve control is introduced. In this approach, the droop curve controlling the ESS is adapted, depending on the ESS' state of charge (SoC), which results in a droop curve with a hysteresis. This concept realizes the charging of the ESS only with recuperation energy, that occurs in the DC microgrid during the production process. Thus, all recuperation energy will be kept in the DC microgrid and a transformation of the energy in $\mathrm{AC}$ or an energy loss through braking resistors will be avoided. Furthermore, no additional energy is needed to charge the ESS. This increases the energy efficiency of the entire production process. The concept was verified in simulation and validated in experiment and it has shown a DC voltage deviation of less than two percent.
\end{abstract}

Index Terms-DC microgrid, Energy efficiency, Uninterruptible power supply, Droop curve control

\section{INTRODUCTION}

The rising awareness of energy efficiency in the industrial sector and the increasing amount of volatile renewable energies makes it necessary to find new concepts for the industrial power supply in the production process. One approach are industrial smart grids and DC microgrids [1], [2]. The motivation of DC microgrids is to achieve lower conversion losses than an AC supply. Moreover all recuperation energy should be completely stored within the DC microgrid [2], to prevent transformation into thermal energy through braking choppers or converting it back to the AC grid. Energy storage systems (ESS) can be used, to store that recuperation energy which occurs during the production process. Another typical application for an ESS is an uninterruptible power supply (UPS). There are several reasons to keep the production running as tools can be destroyed or uncompleted workpieces cannot be finished. UPS also results in a higher reliability of the production process, which is a competitive advantage. Both applications combined - UPS and storing recuperation energy - can increase the synergy potential of ESS, so that the high invest costs can be justified more. For this reason, this paper introduces a failure handling concept for a DC microgrid, which increases the synergy potential of the ESS by having two main purposes: First, to balance differences between energy demand and the energy supply and storing recuperation energy from the production process. Through the recuperation energy the ESS can be charged at no additional costs. Second, in case of an AC grid failure, the energy storage can operate as an UPS to keep the production running.

\section{A. State of the Art}

Architectures and control methods for DC microgrid's load flow management can be distinguished between centralized, decentralized and distributed systems [3]. Centralized method means that all controllable grid participants (i.e. among others a rectifier with boost converter or DC-DC converters) are connected to one central unit. This approach is highly flexible, as the central unit can change operation modes and react on changing conditions online [3]. The disadvantage is a high reactive time and the high reliability requirements for the central control unit. Furthermore, the computational effort of the central unit and the mandatory real-time communication, which is necessary for this architecture, limits the number of participants in DC microgrids.

The decentralized droop curve control is a commonly used approach in many DC microgrid applications, like DC-powered ships or server data centers [4], [5]. The droop curve control is a method to control the grid participants output impedance virtually [6]. In other words, the droop curve can be seen as a virtual impedance of the sources (i.e. the controllable participants). Due to neglecting transmission line impedances, the voltages are equal across the DC microgrid. Based on this assumption, the loads within the DC microgrid are shared among the sources in a reciprocal ratio to their virtual impedance [6]. In consequence, if the load in the DC microgrid increases, the voltage in the grid will decrease but the current output of the sources increases. The droop curve control does not need any communication across the sources. Each source has a local voltage measurement to adjust the current output with respect to the droop curve. The voltage measurement must be low-pass filtered to prevent an oversensitive reaction of the droop curve control [5]. Although droop curve control has advantages like low costs and complexity, it is not flexible enough in many cases such as on changing constraints, like the volatile supply 
This is the author's version of an article that has been published in the ICIT 2019 proceedings.

Changes were made to this version by the publisher prior to publication.

The final version of record is available at https://dx.doi.org/10.1109/ICIT.2019.8755017

of the PV system or the State of Charge (SoC) of an ESS [3]. For this reason, adaptive droop control approaches also known as distributed control, have been introduced to face the lack of flexibility [3]. In this paper, an adaptive approach is used which is introduced in [2], and in [7] it is analyzed and investigated in simulation. In this method, the droop curve control is combined with an additional communication, that can tune the droop curves online in order to optimize the load flow. Thus the droop curve sizing and adjustment can not only be done prior of production process, but also online during the production process.

In [8], [9] distributed UPS only for AC grids are introduced as well as the UPS standard IEC 62040-3 does only consider AC grids [10]. In [11] an UPS for DC microgrids in commercial buildings is outlined. However, there is only a consideration of the ESS control strategy in case of an emergency. On the contrary, this paper proposes an UPS realization within a DC microgrid with an adaptive droop control algorithm, including one or more multi-purpose ESS. This new concept allows a balancing of energy demand and supply within the DC microgrid in case of no failure. Furthermore, the adaptive droop curve algorithm secures that the ESS always keeps a predefined value of SoC to provide an UPS at any time. In case of a mains supply failure, the droop curve of the ESS maintains the power supply of the DC microgrid.

The remainder of this paper is structured as follows: The modeling of the DC microgrid, the ESS and the droop curve control is outlined in Section II. The concept of droop curve sizing and the failure handling are explained in Section III. Measurement and validation results are shown in Section IV. The conclusion follows in Section V.

\section{System Description AND Modeling}

In order to show the functionality of this concept, a fictitious industrial DC microgrid is introduced, which contains mandatory as well as optional components. Fig. 1 shows the structure of the industrial DC microgrid. The DC microgrid is coupled to the AC grid through an unidirectional AC-DC rectifier with a boost converter. Furthermore, a lithium-ion battery is integrated as an ESS and a photovoltaic system (PV). Both, the ESS and the PV system, are connected with DC-DC converters to the DC microgrid. In this paper, the consumers are considered as four different robots, which are part of production process.

\section{A. DC Microgrid Modeling}

The DC microgrid is modeled as a simplified electric network with one capacity $C_{\mathrm{DC}}$ between DC terminals, as shown in Figure 2. According to the Kirchhoff's current law the sum of the participants' currents $i_{n}$ of the DC microgrid are equal to zero, as

$$
\sum_{n} i_{n}=0
$$

At the branch point of the capacity $C_{\mathrm{DC}}$, Eq. (1) is evaluated as follows

$$
i_{\mathrm{C} C}-i_{\mathrm{Rec}}-i_{\mathrm{ESS}}-i_{\mathrm{PV}}-i_{\mathrm{Load}_{1}}, \ldots,-i_{\mathrm{Load}_{4}}=0,
$$

where $i_{\mathrm{Rec}}$ is the rectifier's current, $i_{\mathrm{ESS}}$ and $i_{\mathrm{PV}}$ are the current outputs from the DC-DC converters which control the load flow from the ESS and the PV system, respectively. $i_{\text {Load }_{1}}, \ldots, i_{\text {Load }_{4}}$ are the currents of the four loads. As this model neglects additional transmission line impedances, it can be assumed that the DC grid voltage $u_{\mathrm{DC}}$ is equal to the capacity voltage $u_{\mathrm{c}}$. Due to this simplification, the DC grid voltage $u_{\mathrm{DC}}$ can be obtained by

$$
u_{\mathrm{DC}}(t) \approx u_{\mathrm{C}}(t)=u_{0}+\frac{1}{C_{\mathrm{DC}}} \int_{t_{0}}^{t} i_{\mathrm{c}}(\tau) \mathrm{d} \tau,
$$

where $u_{0}$ is the initial voltage and $t_{0}=0$ the start time and $t$ the current time. However, in the presented approach a discrete-time integration approximation with a small time sample $\Delta t$ is used and applied to Eq. (3), which yields

$$
u_{k+1}=u_{k}+\frac{1}{C_{\mathrm{DC}}} i_{k} \Delta t
$$

where $i_{k}$ is the current and $u_{k}$ the voltage at time step $k$. $u_{k+1}$ is the DC voltage at the following time step $k+1$ and at the first step $k=0$ the voltage $u_{k}$ is equal to the initial voltage $u_{0}$ of Eq. (3). In order to calculate the battery's SoC, the coulomb counting method is used [12]. This means that

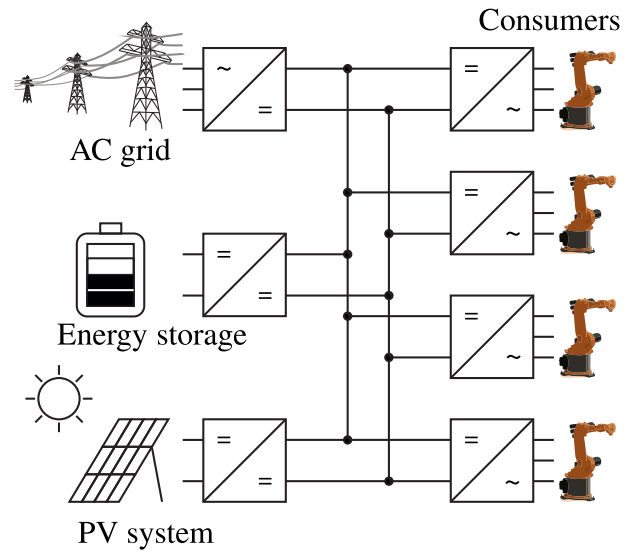

Fig. 1. DC microgrid system

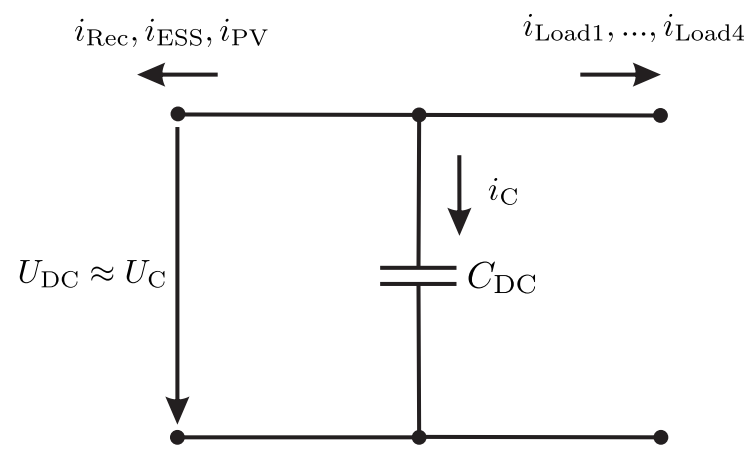

Fig. 2. Model of the DC microgrid. 
This is the author's version of an article that has been published in the ICIT 2019 proceedings.

Changes were made to this version by the publisher prior to publication.

The final version of record is available at https://dx.doi.org/10.1109/ICIT.2019.8755017

the actual battery's current $i_{\text {bat }}$ is integrated over time which yields

$$
s(t)=s_{0}+\int_{t_{0}}^{t} \frac{\eta i_{\text {bat }}(\tau)}{Q} \mathrm{~d} \tau .
$$

The current SoC is $s(t)$ and $s_{0}$ is the initial SoC. $\eta$ is the charge and discharge efficiency of the battery and $Q$ is the maximum capacity of the battery cells in amperehours. The same transformation to a discrete-time integration approximation is applied to Eq. (5) giving

$$
s_{k+1}=s_{k}+\frac{\eta_{\text {bat }} i_{\text {bat }, k}}{Q} \Delta t .
$$

$s_{k+1}$ is the SoC in the following time step and $s_{k}$ the SoC in the current step. $\Delta t$ is the same time sample as used in Eq. (4). The DC microgrid and the battery are coupled by the DC-DC converter, as they have different voltage levels. Hence, $i_{\text {ESS }}$ on DC microgrid side has to be converted to a current on battery side $i_{\text {bat }}$, by using the power equilibrium. This means that the power flow on DC microgrid side $P_{\mathrm{DC}, \mathrm{ESS}}=u_{\mathrm{DC}} i_{\mathrm{ESS}}$ is equal to the power flow on battery side $P_{\mathrm{bat}}=U_{\mathrm{bat}, \mathrm{nom}} i_{\mathrm{bat}}$. However this assumption is only valid, if all power losses and dynamics of the DC-DC converter are neglected. Thus, $i_{\text {bat }}$ can be calculated, by

$$
i_{\mathrm{bat}}=\frac{i_{\mathrm{ESS}} u_{\mathrm{DC}}}{U_{\mathrm{bat}, \mathrm{nom}}} .
$$

Note that the nominal voltage of the battery $U_{\text {bat,nom }}$ is assumed to be constant to achieve a lower computational effort. This is a justified simplification as the battery, which is used for the experiments in Section IV, showed negligible voltage changes for a small depth of discharge (DoD).

\section{B. PV and Load Profile}

The load profiles of the DC microgrid's robots are generated with a model, which is introduced in [13]. There are two different production cycles with $180 \mathrm{~s}$ length each cycle and $20 \mathrm{~ms}$ resolution. This load profile is repeated five times, resulting in a total length of $1800 \mathrm{~s}$. The total power load $P_{\text {total }}=P_{1}+\ldots+P_{4}$ of the four robots are illustrated in Fig. 3 (a) and in Fig. 3 (b) the PV profile is illustrated. Each load and the PV profile are transformed into a current value by

$$
i_{\triangle}=\frac{P_{\triangle}}{u_{\mathrm{DC}}} \text { with } \triangle \in\{\operatorname{Load} 1, \cdots, \operatorname{Load} 4, \mathrm{PV}\}
$$

Note that the PV profile is a $24 \mathrm{~h}$ measurement result from a real plant. This profile was shrunk to $1800 \mathrm{~s}$ to have the same length as the production cycle. That procedure has two reasons: First, one duration can be kept at $1800 \mathrm{~s}$, which is necessary to perform simulations and experiments in an appropriate time frame. Second, there is a high variety of the sum of all loads $P_{\text {sum }}=P_{1}+\ldots+P_{4}+P_{\mathrm{PV}}$ within the length of $1800 \mathrm{~s}$. This variety of $P_{\text {sum }}$ is necessary to proof the approach of the paper for different conditions.

\section{Droop Curve Computation}

The droop curve computation is realized by a linear interpolation. First $u_{\mathrm{DC}}$ is low-pass filtered to $\overline{u_{\mathrm{DC}}}$ [5] and then interpolated by using a look-up table. Then a general description of the linear interpolation yields

$$
i_{\triangle}=f_{\triangle}\left(\overline{u_{\mathrm{DC}}}\right) \text { with } \triangle \in\{\text { Rec, ESS, PV }\} .
$$

In case of the ESS, the droop curve has the SoC as an additional dependency $i_{\mathrm{ESS}}=f_{\mathrm{ESS}}\left(\overline{u_{\mathrm{DC}}}, S o C\right)$. A detailed description of the droop curve's SoC dependency is shown in the next chapter.

\section{Droop Curve Sizing \& FAILURE HANDling CONCEPT}

\section{A. Requirements}

For the suitable droop curve control dimensioning, it has to be taken into account that all recuperation energy from the robots has to be kept within the DC microgrid, as there is no bidirectional AC-DC converter. Moreover the grid must stay in operation for a predefined time in case of an AC grid blackout. From these requirements several preconditions for the droop curves can be derived:

First, the mains supply provides the mean power load of the grid and the lower and upper voltage limits of the devices must not be exceeded. Second, the ESS is supposed to store all recuperation energy from the production process as well as it supports peak loads. Third, all renewable energy sources provide their maximum power output. Fourth, the ESS must not exceed a certain lower SoC limit. This SoC limit is dependent on the amount of energy, which has to be available in case of an mains supply failure. Fifth, as the production
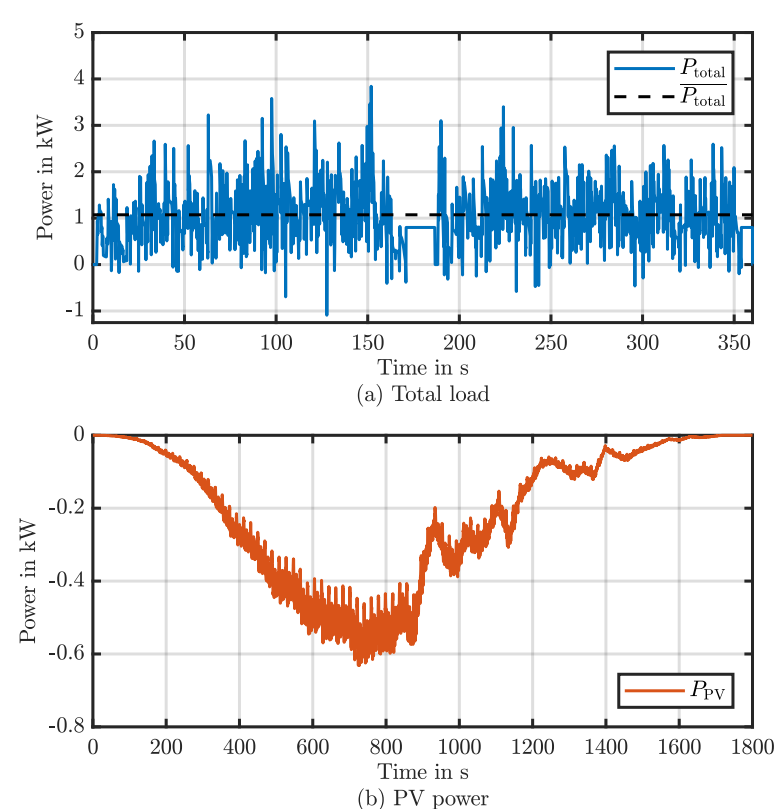

Fig. 3. The loads of the consumers and the PV system. (a) Total load $P_{\text {total }}$ and mean load $\overline{P_{\text {total }}}$; (b) $\mathrm{PV}$ power $P_{\mathrm{PV}}$ 
This is the author's version of an article that has been published in the ICIT 2019 proceedings.

Changes were made to this version by the publisher prior to publication.

The final version of record is available at https://dx.doi.org/10.1109/ICIT.2019.8755017

cylce is changing, the load cycle is changing as well. Hence, the droop curves cannot be optimized to one specific load cycle. However, the ESS should maintain at a constant SoC on average. This ensures that the ESS is always able to absorb recuperation energy and that there is enough SoC for a mains supply failure. Sixth, the DoD of ESS should be kept low. For many ESS such as Lithium-Ion batteries the lifetime strongly depends on the DoD [14]. In standard condition the DoD range should be less than $10 \%$.

\section{B. Droop Curve Sizing}

Under the preconditions mentioned above, the droop curves can now be dimensioned. The lower voltage limit is $U_{\text {min }}=600 \mathrm{~V}$ as it is the lower voltage peak value of the $\mathrm{AC}$ input voltage with an additional safety factor. The upper limit is device-specific as $U_{\max }=800 \mathrm{~V}$, [15], [16]. Due to these boundaries, the nominal voltage is set to the center of the voltage band, which is $U_{\text {nom }}=700 \mathrm{~V}$. As the mains supply provides the mean power demand of the robots $\overline{P_{\text {total }}} \approx 1 \mathrm{~kW}$ (Figure 3 (a)), the current output for the mains supply can be set as

$$
i_{\text {nom }}=\frac{P_{\text {mean }}}{U_{\text {nom }}} .
$$

The maximum of $P_{\text {total }}$ is around $P_{\max } \approx 4 \mathrm{~kW}$. Thus, the rectifier maximum output is set to $P_{\text {rec,max }}=3 \mathrm{~kW}$ at $600 \mathrm{~V}$, which does not cover the complete load, because the ESS is always supposed to support the rectifier in case of a peak load. Otherwise, the ESS would never discharge on average but only charge on average, as the recuperation energy completely flows to the ESS. The minimum power of the rectifier is set to $P_{\text {rec, } \min } \approx 0 \mathrm{~kW}$ at $725 \mathrm{~V}$, as can be seen in Fig. 4 (a). This voltage is set to avoid changing gradients in the mains supply droop curve. Note that the sign convention is defined as follows: negative current means that the current enters the DC microgrid and positive currents leave the grid.
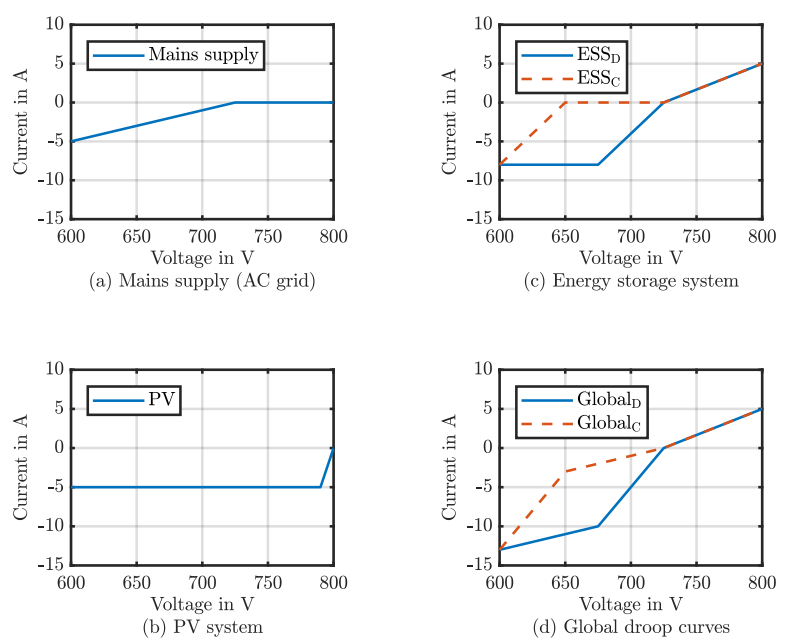

Fig. 4. Droop curves of all sources. (a) Rectifier; (b) PV; (c) ESS $\mathrm{D}_{\mathrm{D}}$ and ESS $_{\mathrm{C}}$; (d) Global $\mathrm{D}$ and Global $_{\mathrm{C}}$
For a maximum energy extraction of the PV, a standard procedure can be used like a maximum power point tracking (MPPT) [5]. The PV load will be only limited if the maximum current of the DC-DC converter is exceeded or in case of a $u_{\mathrm{DC}}$ exceedance. In Fig. 4 (b) the PV droop curve is shown. From $600 \mathrm{~V}$ to $790 \mathrm{~V}$ the droop curve is limited to the maximum current of the DC-DC converter. From $790 \mathrm{~V}$ to $800 \mathrm{~V}$ the $\mathrm{PV}$ power input will be linearly decreased to zero to prevent excess voltage in the DC microgrid.

\section{Energy Storage Droop Curve Algorithm}

In this approach, the DC-DC converter of the ESS has an adaptive droop curve in order to keep the ESS at a constant SoC on average. As it is possible that the load cycle or the PV load input changes by time, the droop curve cannot be exactly dimensioned for a specific load cycle to keep an average SoC. For this reason we have a first droop curve $\mathrm{ESS}_{\mathrm{D}}$ with a primarily discharging behavior, and a second droop curve ESS $_{C}$ with a primarily charging behavior combined with a droop curve switching algorithm. In case of $\mathrm{ESS}_{\mathrm{D}}$, the energy storage supports the DC microgrid more than in case of $\mathrm{ESS}_{\mathrm{C}}$. The recuperation energy is completely absorbed by the ESS in any case. Both ESS droop curves must be designed so that $P_{\max }=4 \mathrm{~kW}$ and $P_{\min }=-1 \mathrm{~kW}$ load can be covered by ESS completely. This is necessary to assure that the ESS can supply the DC microgrid self-sufficient in case of an AC grid blackout. Otherwise, the ESS might not maintain the DC voltage at peak loads of $P_{\text {total }}$. ESS $\mathrm{DS}_{\mathrm{D}}$ and $\mathrm{ESS}_{\mathrm{C}}$ are visualized in Fig. 4 (c). Both curves cover $P_{\max }$ at $600 \mathrm{~V}$ and $P_{\min }$ at $800 \mathrm{~V}$. However ESS $_{\mathrm{C}}$ contains a zero ampere plateau between $650 \mathrm{~V}$ and $725 \mathrm{~V}$ compared to $\mathrm{ESS}_{\mathrm{D}}$. Consequently both ESS droop curves perform a hysteresis, which balances the ESS on an average SoC.

The droop curve switching algorithm works as follows: Assuming that the $\mathrm{ESS}_{\mathrm{D}}$ is selected by the DC-DC converter at a certain point in time. At the same moment the $\mathrm{SoC}$ is higher or equal to a defined upper SoC boundary $S o C_{\text {high }}$. The ESS will now discharge on average, while the production process is running. At a defined lower SoC boundary $S o C_{\text {low }}$ the DCDC converter changes the droop curve automatically to the ESS $_{\mathrm{C}}$. The ESS will now charge on average as the time goes by until $S o C_{\text {high }}$ is reached again. Then the process starts over by re-selecting $\mathrm{ESS}_{\mathrm{D}}$. In this paper, the upper SoC boundary is set to $S o C_{\mathrm{high}}=65 \%$ and for the lower SoC boundary the authors recommend $S o C_{\text {low }}=60 \%$. This selection ensures a low depth of discharge, thus a high battery cycle life time. Furthermore, at least $60 \%$ of the SoC is always available in case of an AC grid blackout to keep the DC microgrid alive for a predefined amount of time (UPS-ability). The amount of energy which must be stored to guarantee UPS is not suggested in this paper, as it depends on the application, the load cycle and the size of UPS-period. Hence, the ESS sizing can only be assessed for a specific DC microgrid with specific requirements of the grid operator.

Fig. 4 (d) shows the global curve, which is the superposition of the mains supply and the ESS droop curves. Depending 
This is the author's version of an article that has been published in the ICIT 2019 proceedings.

Changes were made to this version by the publisher prior to publication.

The final version of record is available at https://dx.doi.org/10.1109/ICIT.2019.8755017

TABLE I

Detailed Energy Storage System

\begin{tabular}{c||c|c|c}
\multicolumn{1}{c||}{ Data } & Value & Variable & Unit \\
\hline Type & Lithium-ion battery & & \\
\hline Cell chemistry & $\begin{array}{c}\text { NMC Lithium-ion } \\
\text { polymer }\end{array}$ & & \\
\hline Nominal Voltage & 107 & $U_{\text {bat,nom }}$ & $\mathrm{V}$ \\
\hline Cell Capacity & 100 & $Q$ & $\mathrm{Ah}$ \\
\hline $\begin{array}{c}\text { Max. Power (Limited } \\
\text { by DC-DC converter) }\end{array}$ & 5 & $P_{\max }$ & $\mathrm{kW}$ \\
\hline Efficiency & 98 & $\eta_{\text {bat }}$ & $\%$ \\
\hline
\end{tabular}

on whether $\mathrm{ESS}_{\mathrm{D}}$ or $\mathrm{ESS}_{\mathrm{C}}$ is chosen, there are two different global curves Global $_{D}$ and Global $_{C}$. This visualization helps to see the total possible current at a certain $u_{\mathrm{DC}}$. The PV droop curve is not superimposed, as it is a not controllable source.

\section{EXPERIMENT AND RESULTS}

In order to validate the simulation results, a DC microgrid test rig was set up. The detailed description of the test rig follows in the first part of this section. In the second part, the results of three different experiments performed with this test rig are presented.

\section{A. Test Rig}

The test rig for the experiments contains an AC-DC converter (Fig. $5 I$ ) as the droop curve controlled mains supply. The test rig also contains a DC-DC converter (Fig. $5 I I$ ) coupled with an ESS. This DC-DC converter is controlled by the droop curve as well, including the switching algorithm as mentioned in Section III-C. As ESS, a Lithium-Ion battery with $10 \mathrm{kWh}$ energy stored is used. The detailed battery properties are listed in Tab. I. The overall DC microgrid's capacity is $C_{\mathrm{DC}}=14.2 \mathrm{mF}$, which is conditioned by the device configuration and is used for simulation as well. The motor inverter (Fig. $5 I I I$ ) with the drive (Fig. $5 D_{1}$ ) is used

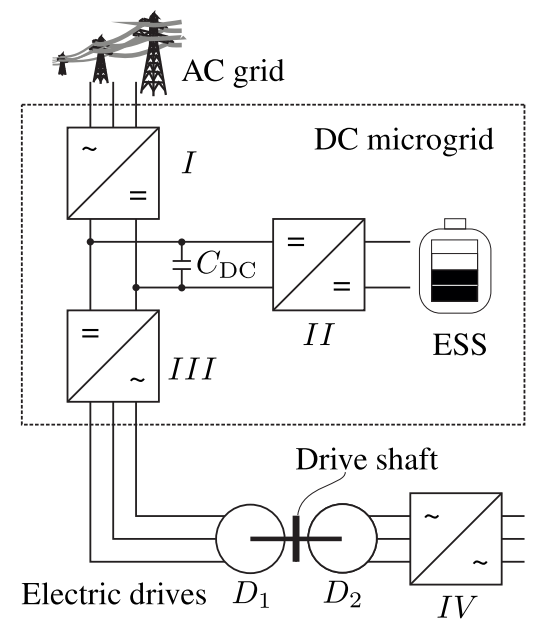

Fig. 5. DC microgrid test rig to represent the sum of all loads $P_{\text {sum }}$ including the load of the four robots $P_{\text {total }}$ (Fig. 3 (a)) and the PV system (Fig. 3 (b)). A second electric drive (Fig. $5 D_{2}$ ), supplied by (Fig. $5 I V$ ), is used to absorb the energy which is released by the load representation. Both drives are mechanically coupled over the drive shafts. In order to run various load profiles, $D_{1}$ is torque controlled and $D_{2}$ is controlled to a constant rotational speed. Assuming that $P_{\mathrm{el}} \approx P_{\text {mech }}$ the torque $M$ for $D_{1}$ can be calculated as

$$
M=\frac{P_{\mathrm{el}}}{2 \pi n}=\frac{P_{\mathrm{sum}}}{2 \pi n} .
$$

The rotational speed $n$ is constant and set to $1800 \mathrm{rpm}$ as that is the most efficient speed for both drives.

\section{B. Results}

Three scenarios with $1800 \mathrm{~s}$ length each containing the load profile and PV profile, as described in Section II-B, were tested in simulation and experiment. Scenario I is a standard situation without any AC grid blackout. Scenario II contains an AC grid blackout, which occurs at $900 \mathrm{~s}$ and remains until the end of the scenario. Scenario III starts similar to Scenario II with an AC grid blackout at $900 \mathrm{~s}$, and additionally the AC grid returns to standard operation at $1500 \mathrm{~s}$. The experiments were started with $65 \%$ initial SoC and started with droop curve $\mathrm{ESS}_{\mathrm{D}}$. Note that in order to proof the concept the lower SoC boundary of the ESS droop curve is set to $S o C_{\text {low }}=64 \%$ instead of $S o C_{\text {low }}=60 \%$. Otherwise, a scenario length of $1800 \mathrm{~s}$ is too short to observe an adjustment of the ESS droop curves.

1) Validation: Figure 6 shows the comparison of simulation and experiment for a zoom in the time range of $10 \mathrm{~s}$ to $20 \mathrm{~s}$ of Scenario I. However, these results can be also considered for the other scenarios, as all three scenarios started with the same conditions and the AC grid blackout in Scenario II and Scenario III occurs later. Figure 6 (a) is the load profile including the PV profile and it shows that there is a good match between simulation and experiment. The power output of the rectifier is shown in Figure 6 (b). There is an offset between simulation and experiment because of the missing power loss in the simulation model. Consequently the Normalizedroot-mean-square deviation of the model's power prediction from the experiment is up to $17 \%$. Figure 6 (c) shows the power curve of the battery. The power support to the grid is significantly high, which is a consequence of $\mathrm{ESS}_{\mathrm{D}}$ droop curve, which is selected at this point in time. The oscillation from $17 \mathrm{~s}$ to $18 \mathrm{~s}$, is caused by the droop curve's upper break point close to $u_{\mathrm{DC}} \approx 725 \mathrm{~V}$. At this point the current switches often between $i_{\mathrm{ESS}}=0$ and $i_{\mathrm{ESS}} \neq 0$. Figure $6(\mathrm{~d})$ visualizes the $u_{\mathrm{DC}}$ and the results show a good qualitative match between simulation and experiment. However, there is a significant voltage ripple in the experiment being a consequence of the rectifier's PWM control $\left(f_{\mathrm{PWM}}=4.2 \mathrm{kHz}\right)$.

2) Scenario I: In Figure 7 the voltage $u_{\mathrm{DC}}$ for all three scenarios are shown for the entire duration of the scenarios (1800 s). The voltage curve of Scenario I (Figure 7 (a)) shows a higher voltage fluctuation from a certain point in time. At this 
This is the author's version of an article that has been published in the ICIT 2019 proceedings.

Changes were made to this version by the publisher prior to publication.

The final version of record is available at https://dx.doi.org/10.1109/ICIT.2019.8755017

point in time the ESS droop curve has been adjusted, because the lower SoC boundary has been reached $\left(S o C_{\text {low }}=64 \%\right)$. After that, the voltage fluctuation is significantly higher, due to the zero ampere plateau between $650 \mathrm{Vand} 750 \mathrm{~V}$ of $\mathrm{ESS}_{\mathrm{C}}$. In simulation the droop curve adjustment occurs at $1036 \mathrm{~s}(*$ in the zoom of Figure 7 (a)) and in experiment at $1154 \mathrm{~s}(* *$ in the zoom of Figure 7 (a)). The results show that the lower SoC boundary is reached with a slight time difference of around $100 \mathrm{~s}$. The time difference is a consequence of the inaccurate SoC assessment which is provided by ESS. On the other hand, the consumption of energy is low $(<200 \mathrm{Wh})$ compared to the amount of energy stored $\left(E_{\mathrm{bat}} \approx 10 \mathrm{kWh}\right)$ in the ESS. For
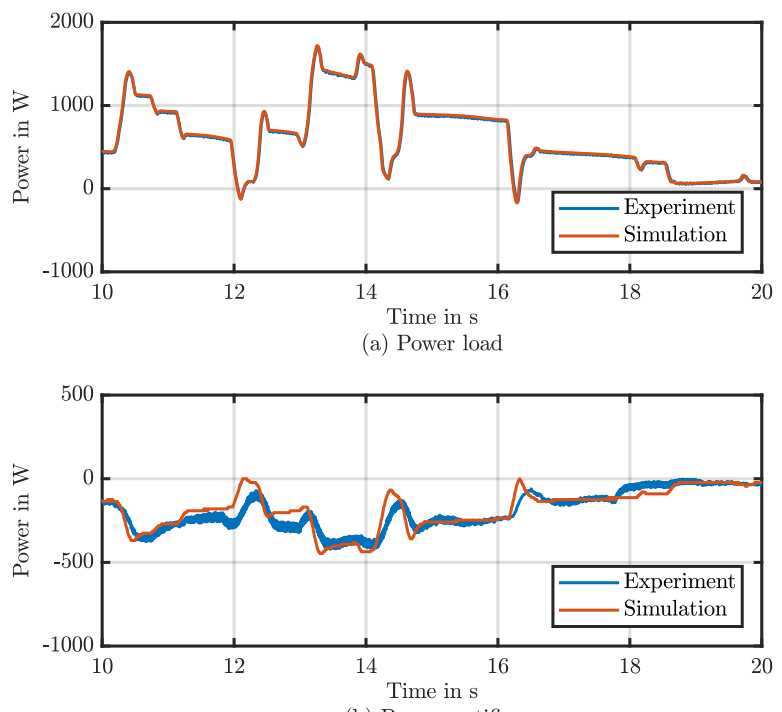

(b) Power rectifier

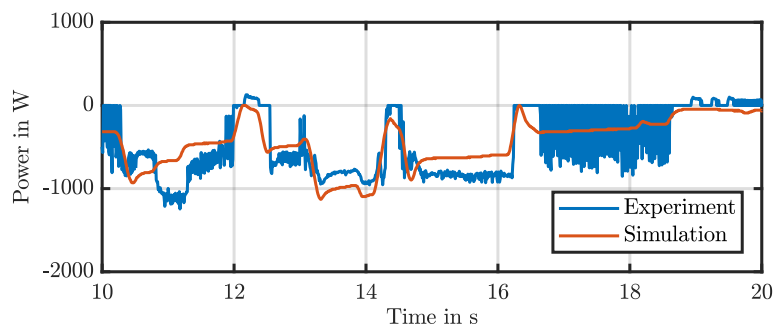

(c) Power battery

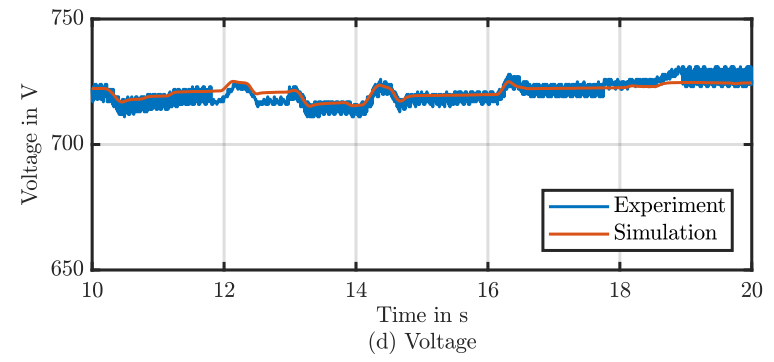

Fig. 6. Zoom from $10 \mathrm{~s}$ to $20 \mathrm{~s}$ for all scenarios. (a) Power load, (b) Power rectifier, (c) Power DC-DC converter coupled with ESS, (d) Voltage this reason, the discharge of one percent SoC takes a long time. Even small differences in the power consumption between simulation and experiment can lead to significant differences of the SoC assessment.

3) Scenario II: In Scenario II (Figure 7 (b)) the $\mathrm{AC}$ grid blackout occurs at $900 \mathrm{~s}$. First, the droop curve $\mathrm{ESS}_{\mathrm{D}}$ maintains the voltage level until the lower SoC boundary has been reached. This happens around $935 \mathrm{~s}$ in simulation and $937 \mathrm{~s}$ in experiment, as can be seen in the zoom of Figure 7 (b) on the right. After this time the ESS droop curve is adjusted to $\mathrm{ESS}_{\mathrm{C}}$. Figure 8 shows the energy consumption of the ESS (a) and the SoC curve (b) of Scenario II. After the mains supply failure, the energy consumption significantly increases, as the whole DC microgrid is only supplied by the battery. The neglection of power losses becomes apparent in a higher energy consumption in experiment than predicted in simulation as the curves in Figure 8 (a) diverge.
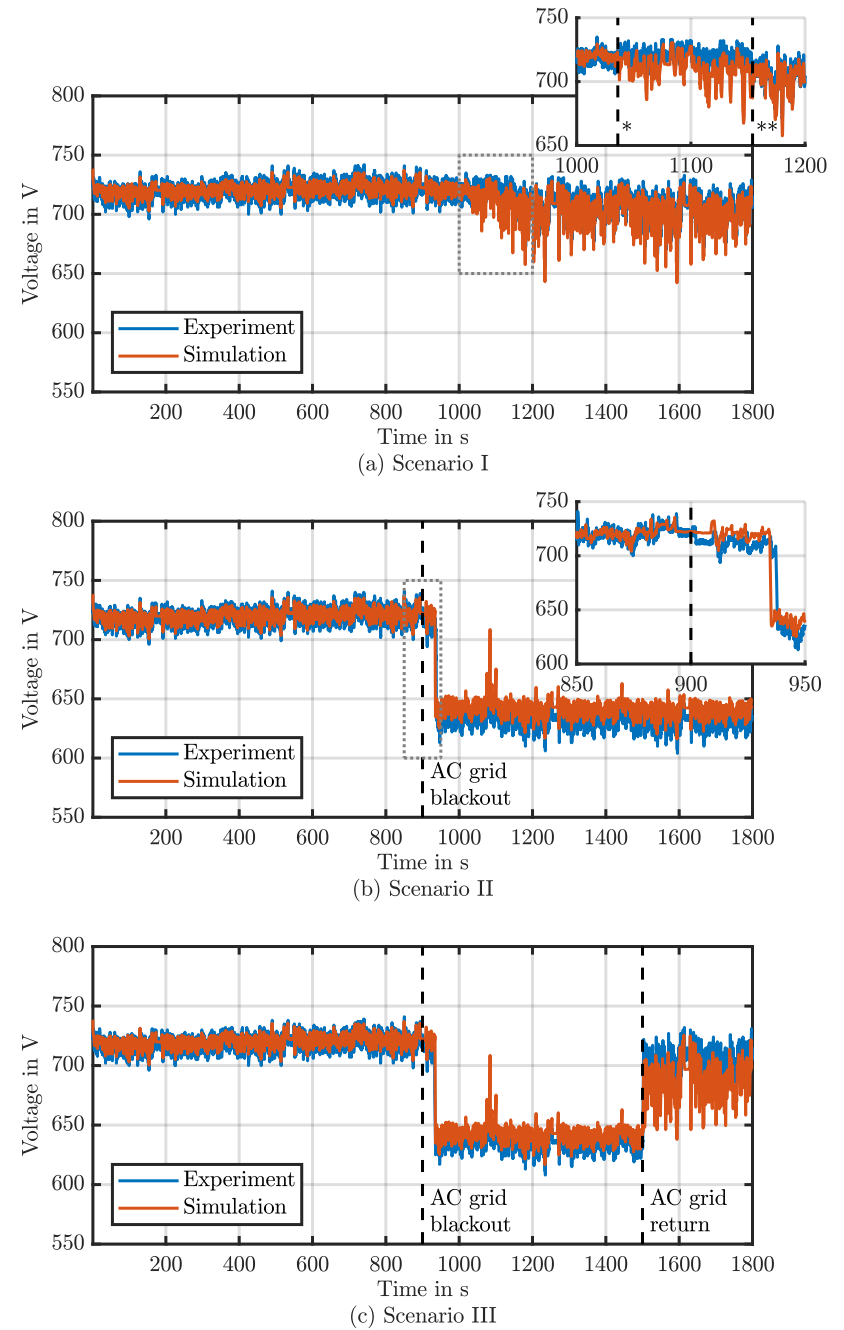

Fig. 7. Voltage curves of all scenarios. (a) Voltage - Scenario I, (b) Voltage - Scenario II, (c) Voltage - Scenario III

Copyright (c) 2019 IEEE. Personal use of this material is permitted. For any other purposes, permission must be obtained from the IEEE by emailing pubs-permissions@ieee.org. 
This is the author's version of an article that has been published in the ICIT 2019 proceedings.

Changes were made to this version by the publisher prior to publication.

The final version of record is available at https://dx.doi.org/10.1109/ICIT.2019.8755017

4) Scenario III: The voltage curve of Scenario III is visualized in Figure 7 (c). In the beginning, the voltage curve is similar to Scenario II with a drop of the voltage shortly after the mains supply failure, due to the ESS droop curve adjustment. From $1500 \mathrm{~s}$, the AC grid returns into operation and the voltage rises, however the voltage fluctuation is higher than before the mains supply failure. This is because, the ESS droop curve remains at $\mathrm{ESS}_{\mathrm{C}}$, which contains the zero ampere plateau, until the end of the experiment.

The results have shown that the approach works successfully. Due to this approach, all recuperation energy is stored within the DC microgrid, as neither a braking chopper nor bidirectional AC-DC converter was used. In case of a $\mathrm{AC}$ grid blackout, the method successfully keeps the entire DC microgrid in operation.

\section{CONCLUSION}

The industrial DC microgrid with droop curve control can increase the energy efficiency in production. Renewable energies as well as ESS can be integrated easily. This paper has shown, that an AC grid failure can be handled with an ESS in order to realize an UPS. The DC microgrid maintains its full functionality while the AC grid blackout occurs. Charging of the ESS is realized from recuperation of the production process (e.g. deceleration of drives). Thus, no braking chopper are needed anymore, which makes the DC microgrid more efficient and it increased the synergy potential of the ESS. The droop curve sizing of the ESS was implemented in a way that the ESS is always charged by recuperation energy. Furthermore the ESS's droop curve is dependent on the SoC. When the SoC reaches a higher or lower boundary, the droop curve will be adjusted, so that the $\mathrm{SoC}$ is always kept within a certain boundary.
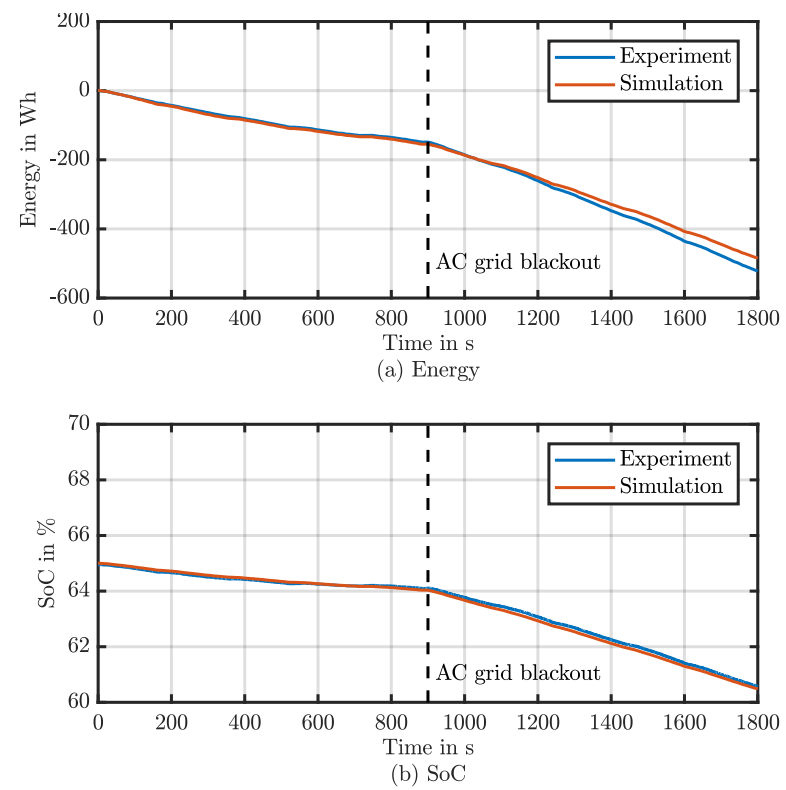

Fig. 8. Scenario II. (a) Energy consumption ESS, (b) SoC of ESS
However, under certain conditions the adjustment of droop curves might lead to an instability of the droop curve control. Therefore, more investigations on the stability of this droop curve adjustment must be done. Furthermore, the paper does not take a consideration on optimal ESS size and costs for an UPS in a DC microgrid into account. Both investigations should be part of future works.

\section{ACKNOWLEDGMENT}

This project was part of the DC-INDUSTRIE research project, funded by the German Federal ministry of Economic Affairs and Energy (BMWi).

For further information see: www.dc-industrie.de

\section{REFERENCES}

[1] T. Samad and S. Kiliccote, "Smart grid technologies and applications for the industrial sector," Computers \& Chemical Engineering, vol. 47, pp. 76-84, 2012.

[2] H. Borcherding, J. Austermann, T. Kuhlmann, B. Weis, and A. Leonide, "Concepts for a dc network in industrial production," in 2017 IEEE Second International Conference on DC Microgrids (ICDCM). IEEE, 2017, pp. 227-234.

[3] T. Dragicevic, X. Lu, J. Vasquez, and J. Guerrero, "Dc microgrids-part i: A review of control strategies and stabilization techniques," IEEE Transactions on Power Electronics, vol. 31, no. 7, pp. 4876-4891, 2016.

[4] B. Wunder, L. Ott, J. Kaiser, Y. Han, F. Fersterra, and M. Marz, "Overview of different topologies and control strategies for dc micro grids," in 2015 IEEE First International Conference on DC Microgrids (ICDCM). IEEE, 2015, pp. 349-354.

[5] L. Ott, Y. Han, B. Wunder, J. Kaiser, F. Fersterra, M. Schulz, and M. Marz, "An advanced voltage droop control concept for grid-tied and autonomous dc microgrids," in 2015 IEEE International Telecommunications Energy Conference (INTELEC). IEEE, 2015, pp. 1-6.

[6] V. Nasirian, A. Davoudi, and F. L. Lewis, "Distributed adaptive droop control for dc microgrids," in 2014 IEEE Applied Power Electronics Conference and Exposition - APEC 2014. IEEE, 2014, pp. 1147-1152.

[7] D. A. Schaab, S. Weckmann, T. Kuhlmann, and A. Sauer, "Simulative analysis of a flexible, robust and sustainable energy supply through industrial smart-dc-grid with distributed grid management," Procedia CIRP, vol. 69, pp. 366-370, 2018.

[8] J. M. Guerrero, L. Hang, and J. Uceda, "Control of distributed uninterruptible power supply systems," IEEE Transactions on Industrial Electronics, vol. 55, no. 8, pp. 2845-2859, 2008.

[9] J. M. Guerrero, J. C. Vasquez, J. Matas, M. Castilla, and L. G. de Vicuna, "Control strategy for flexible microgrid based on parallel line-interactive ups systems," IEEE Transactions on Industrial Electronics, vol. 56, no. 3, pp. 726-736, 2009

[10] IEC International Electrotechnical Commission, Uninterruptible power systems (UPS) - Part 3: Method of specifying the performance and test requirements, IEC Std. 62 040-3, 2011.

[11] S. Hong, S. Cho, K. Kang, and C. Won, "The integrated operation method of emergency generator system for commercial building applied dc distribution system," in 2017 20th International Conference on Electrical Machines and Systems (ICEMS), 2017, pp. 1-6.

[12] G. L. Plett, "Extended kalman filtering for battery management systems of lipb-based hev battery packs: Part 2. modeling and identification," Journal of Power Sources, vol. 134, no. 2, pp. 262-276, 2004.

[13] K. Eggers, E. Knöchelmann, S. Tappe, and T. Ortmaier, "Modeling and experimental validation of the influence of robot temperature on its energy consumption," in 2018 IEEE International Conference on Industrial Technology (ICIT), 2018, pp. 239-243.

[14] H. Wenzl, "Batteries and fuel cells - lifetime," in Encyclopedia of Electrochemical Power Sources. Elsevier, 2009, pp. 552-558.

[15] Bosch Rexroth AG, Rexroth IndraDrive ML Universal Inverters HMU05: Instruction Manual, Lohr am Main, 2017. [Online]. Available: http://www.boschrexroth.com/various/utilities/mediadirectory

[16] Bosch Rexroth AG, IndraDrive Power Supply Basic PSB-20 Functions. Application Manual, Lohr am Main, 2017. [Online]. Available: http://www.boschrexroth.com/various/utilities/mediadirectory 